\title{
Occult mucin-producing cholangiocarcinoma in situ: a rare clinical case with difficult tumour staging
}

\author{
Muneyasu Kiriyama, Tomoki Ebata, Yukihiro Yokoyama, Tsuyoshi Igami, Gen Sugawara, Takashi Mizuno,
} Junpei Yamaguchi and Masato Nagino*

\begin{abstract}
Background: Mucin-producing cholangiocarcinoma (MPCC) is an uncommon tumour that is clinically characterized by mucin-hypersecretion. Because the initial symptoms of MPCC may be attributed to the viscus mucobilia, the primary tumour mass may potentially be unrecognizable. We report an interesting case of curatively resected occult MPCC in situ.

Case presentation: A 70-year-old man was referred to our hospital with increased levels of biliary enzymes. Multidetector row computed tomography (MDCT) demonstrated a diffuse dilatation of the entire biliary system without evidence of tumour mass. Additionally, there were numerous variably sized cysts throughout the liver. The cyst of S4 was the largest, followed by that of S1, which connected with the right hepatic duct. Endoscopic retrograde cholangiography showed intrabiliary mucus, predominantly in the left hepatic duct, but failed to show a communication of both cysts with the bile duct. We clinically suspected that minute MPCC was present within the S1 cyst and performed left hepatectomy, caudate lobectomy, and resection of the extrahepatic bile duct. Macroscopically, papillary adenocarcinoma in situ was present in the S1 cyst, and a final diagnosis of MPCC originating from the bile duct of the caudate lobe was made.

Conclusions: For MPCC, in practice, we should consider the possibility that this tumour can be occult. In this complicated setting, demonstrating the communication to the responsible dilated duct is a clue to the diagnosis. Multidirectional MDCT images succeeded in specifically demonstrating this communication, which is insensitive to the presence of excessive mucobilia.
\end{abstract}

\section{Background}

Some cholangiocarcinomas secrete excessive mucus into the biliary system [1-4], causing dilatation of the entire biliary system and enlargement of the orifice at the papilla of Vater. These clinical features are uncommon in ordinary cholangiocarcinoma; therefore, this form of cholangiocarcinoma is clinically termed mucin-producing cholangiocarcinoma (MPCC) [1, 4, 5]. Associated symptoms are attributed to the viscous mucobilia rather than pathological obstruction of the bile duct. Therefore, the primary tumour may be potentially minute or unrecognizable if the tumour secretes excessive mucus $[2,3,6,7]$.

Here, we report a rare case with occult MPCC in situ that was resected with tumour-free margins. Lack of radiological

\footnotetext{
* Correspondence: nagino@med.nagoya-u.ac.jp

Division of Surgical Oncology, Department of Surgery, Nagoya University Graduate School of Medicine, 65 Tsurumai-cho, Showa-ku, Nagoya 466-8550, Japan
}

\section{Case presentation}

A 70-year-old man had visited a local physician and had increased levels of biliary enzymes at medical check-up: serum aspartate aminotransferase (72 IU/l), alanine aminotransferase $(212 \mathrm{IU} / \mathrm{l})$, alkaline phosphatase (555 IU/l), gamma glutamyl transpeptidase (218 IU/l), and total bilirubin $(0.4 \mathrm{mg} / \mathrm{dL})$. He had undergone open cholecystectomy 23 years ago. Magnetic resonance cholangiopancreatography (MRCP) revealed a diffuse dilatation of the entire biliary system and bilateral multiple cysts in the liver (Fig. 1). He was referred to our hospital for further evaluation.

Multidetector row computed tomography (MDCT) revealed extensive dilatation of the entire bile duct without evidence of tumour mass or stricture. Among 


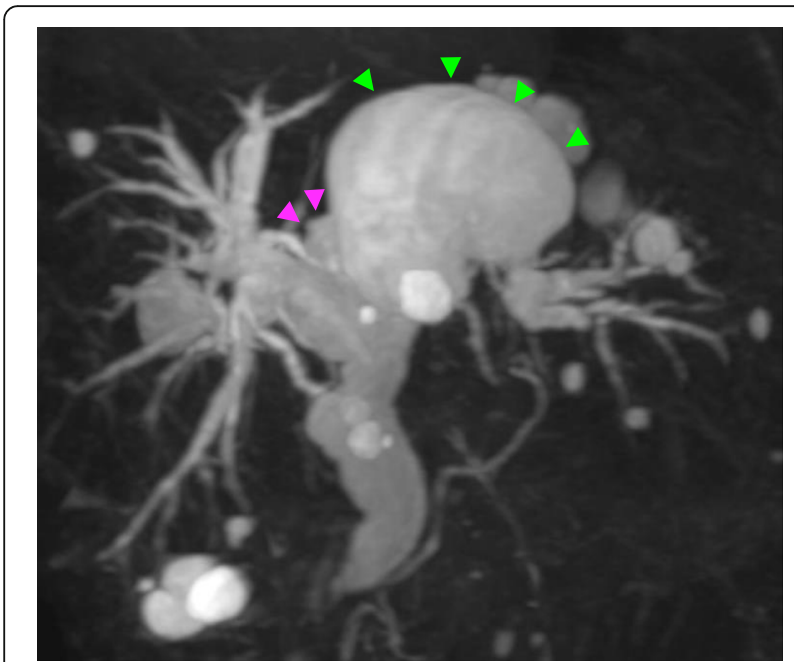

Fig. 1 Magnetic resonance cholangiopancreatography. Multiple liver cysts, including the S1 cyst (pink arrow head) and the S4 cyst (green arrow head), were found. The whole bile duct was extensively dilated

the liver cysts, there were two major adjacent cysts in $\mathrm{S} 1$ (33.1 $\mathrm{mm}$ in size) and $\mathrm{S} 4$ (74.7 $\mathrm{mm}$ in size). The former was evidently connected with the right hepatic duct, whereas the latter was not (Fig. 2). Duodenoscopy revealed a dilated orifice of the Vater's papilla with mucus, indicating the mucin-hypersecreting nature of the disease (Fig. 3a). Endoscopic retrograde cholangiography (ERC) demonstrated mobile ragged translucencies (i.e., intrabiliary mucus), predominantly in the left hepatic duct. ERC failed to demonstrate a communication between the cysts and the bile duct (Fig. 3b). The four biopsy samples taken from the right, left, hilar, and upper bile ducts were all negative for cancer.

The above radiological findings strongly suggested the presence of minute/occult MPCC. For the surgical management, we focused on two radiologic findings: first, the S1 cyst connected to the bile duct, suggesting a primary diseased site; second, mucus was found predominantly in the left hepatic duct, suggesting that the largest S4 cyst should also be resected. Therefore, we planned to perform left hepatectomy, caudate lobectomy, and extrahepatic bile duct resection with dissection of regional lymph nodes. During surgery, because the two cysts adhered to the middle hepatic vein, liver transection was carefully advanced to avoid rupture. We resected the two cysts en bloc without explosion. The operation time was $484 \mathrm{~min}$, and blood loss was $913 \mathrm{ml}$. The patient's postoperative course was uneventful, except for minor bile leakage, and he was discharged 16 days after the surgery.

Macroscopically, the S1 cystic lesion, filled with mucus, connected with the right hepatic duct via the two bile ducts of the right caudate lobe (B1r), the luminal surface of which showed a fine granular pattern (Fig. 4a, b). Microscopically, papillary projection into the lumen, formed of atypical epithelial cells with multilayering nuclei, was found, indicating papillary adenocarcinoma in situ (Fig. 5). A papillary to flat lesion with variable degrees of cellular atypia spread to the B1r. All regional lymph nodes were negative for cancer. According to the American Joint Committee on Cancer [8], the tumour was labeled as stage 0 (TisN0M0) disease. The S4 cyst was a simple cyst without neoplastic epithelium. All margins were tumour-free. Definitive diagnosis of MPCC in situ developing from the B1r was made. The patient remains well without tumour recurrence 3 years and 9 months after the surgery.

\section{Discussion}

MPCC was first reported by Chou et al. [9] in 1976; thereafter, the disease concept has been described by

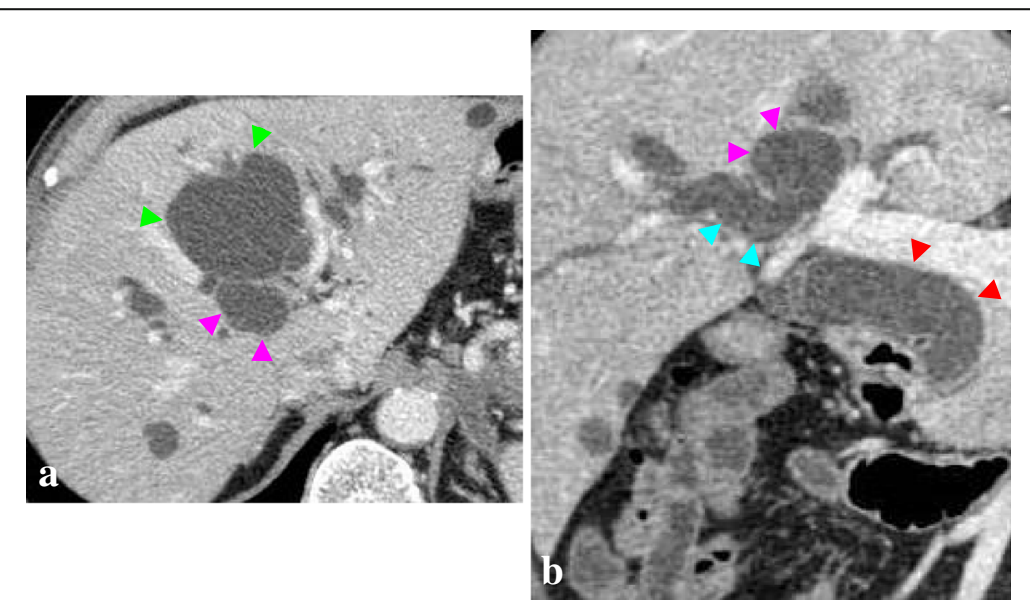

Fig. 2 Multidetector row computed tomography. The S1 cyst (pink arrow head) connected with the right hepatic duct (blue arrow head). The extrahepatic bile ducts were diffusely dilated (red arrow head). The tumour mass, stricture, and wall thickness were not evident in either cyst or the entire bile duct. The green arrow head shows the $\$ 4$ cyst. a Axial image. b Coronal image 

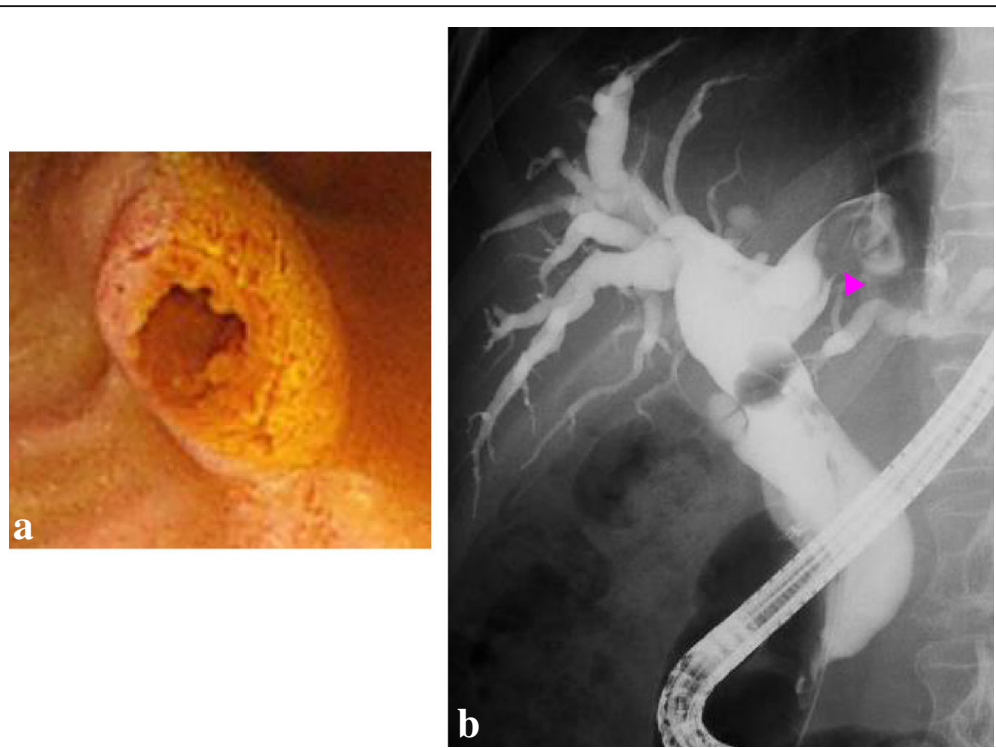

Fig. 3 a. Duodenoscopy. The orifice of the Vater's papilla was expanded by mucus. b. Endoscopic retrograde cholangiography. The entire bile duct was diffusely dilated. There was floating mucus in the left hepatic duct (pink arrow head)
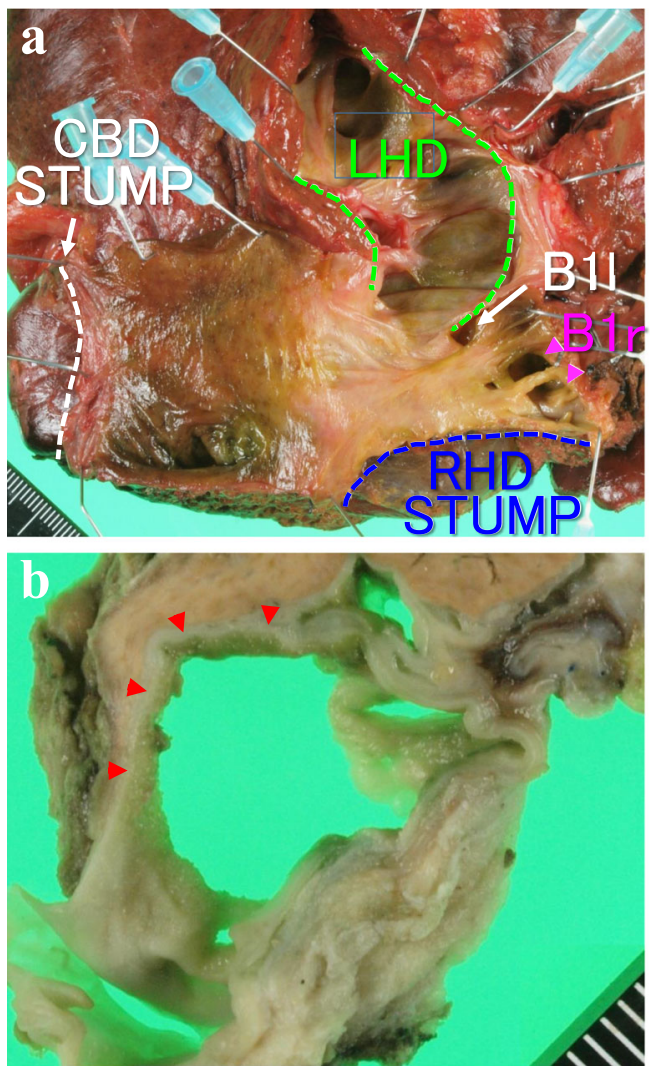

Fig. 4 Macroscopic findings. a. The S1 cystic lesion connected with right hepatic duct via the two bile ducts of the right caudate lobe (pink arrow head). (CBD common bile duct, LHD left hepatic duct, RHD right hepatic duct, $B 1 /$ confluence of the left caudate branch, $B 1$ r: confluence of the right caudate branch). $\mathbf{b}$. Cut surface of the S1 cyst. Fine granular tumour spreads in the luminal surface (red arrow head)
Nagino et al. [1]. MPCC is not a pathological but a clinical disease entity characterized by mucin-hypersecretion, although its extent has not been standardized. Several investigators verified that MPCC histologically exhibits an intraductal papillary proliferation along with delicate fibrovascular cores; heterogenous atypia of the tumour cells; and frequent flat intraepithelial lesion around the primary tumour [10, 11]. These features suggest that MPCC is an overlapping disease category with intraductal papillary neoplasm of the bile duct (IPNB) [10, 12-14]. In this report, we used the term MPCC in the historical sense for the detection of the disease. Alternative terminology of IPNB was also possible in this patient, although the definition of IPNB has not been consistent.

Yang et al. [6] showed that the extent of mucinsecretion bears no relationship to the atypia of the tumour cells; some mucin-producing tumours are pathologically diagnosed as adenoma. Onoe et al. [15] also reported that pathologically malignant potentials representing size, tumour depth, or nodal status were comparable between papillary cholangiocarcinomas with mucin-secretion and those without. These observations fail to confirm specific pathological features and, in turn, suggest a morphological variation in MPCC. In fact, Yeh et al. [16] retrospectively analyzed cholangiographic findings in 13 patients with histologically proven MPCC, of which 8 patients had a finding of mucobilia without gross tumour. Surprisingly, one-third of patients with MPCC had occult (radiologically invisible) tumours. Overall, the following three clinical lessons should be considered: first, mucobilia is a sentinel sign of MPCC; second, MPCC 


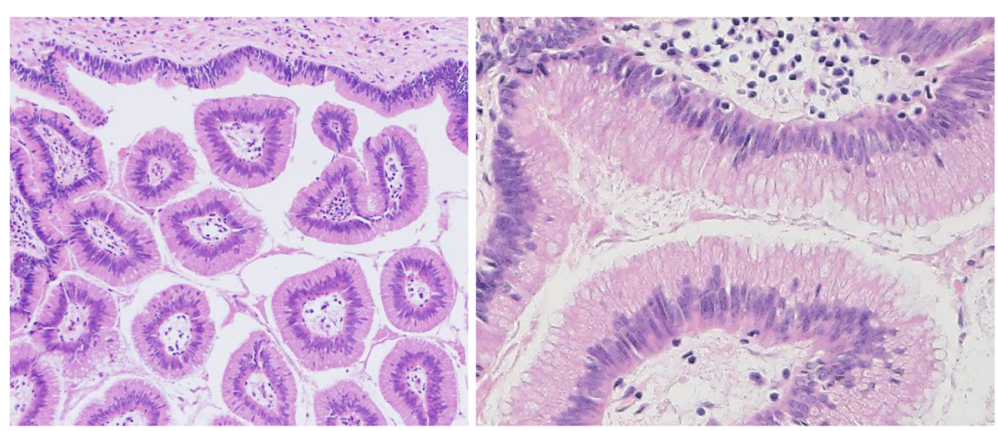

Fig. 5 Histological findings. Papillary projection, which was formed of atypical epithelial cells with multilayering nuclei and moderately increased $\mathrm{N} / \mathrm{C}$ ratio is present in the S1 cyst. Because of the marked papillary structural atypia, despite moderate cellular atypia, the diagnosis of papillary adenocarcinoma in situ was made

exhibits a variety of morphologies; and third, gross neoplasia is unrecognized more frequently than expected. Thus, we should revise our diagnostic strategy to be applicable to patients with a sole sign of mucobilia (occult MPCC).

MPCC exhibits two representative morphologies: ductectatic type and cystic type. The former is defined as a papillary tumour in the dilated intrahepatic bile duct branch that shows disproportional enlargement, and the latter is defined as a papillary tumour within a large liver cyst that communicates with the biliary system [4]. Notably, saturating mucus usually fills not only the diseased cyst but also the communication; therefore, conventional cholangiography has limited value unless specific preparations are performed [17]. In contrast, the presence of mucin never impairs MDCT images, allowing dilated bile ducts to be easily evaluated by multidirectional reconstructions and by paging view on electronic medical records systems. Therefore, MDCT is an important alternative to cholangiography, particularly in occult MPCC.

In the present case, we hypothesized that the occult MPCC was associated with any of the liver cysts (a variation of cystic type MPCC). As the communication between the S1 cyst and the hepatic duct was clearly demonstrated on CT images, the S1 cyst was specified as the target region. Additionally, biopsy samples from the bile duct were all negative for cancer, indicating that the tumour was confined to the cyst and the communication. Based on this limited information, we planned left hepatectomy, caudate lobectomy, and extrahepatic bile duct resection to maximize the chance of $\mathrm{R} 0$ resection.

The clinical impact of the remnant carcinoma in situ at the ductal remains a matter of debate; however, our recent study clearly demonstrates the growing nature of the foci, the risk of local disease relapse, and a deteriorating impact on postoperative survival [18]. These biological behaviours, however, are observed only in patients with early stage cholangiocarcinoma. In another words, ductal margin status is a sole prognostic factor in an early stage of the disease (occult MPCC) [19]. The diagnostic utility of the peroral cholangioscopy (POCS) tool has been reported [5, 20, 21]. POCS provides an accurate diagnostic yield regarding intraepithelial neoplasia in the large bile duct. In this study, we unfortunately did not perform POCS, mainly due to the endoscopists' preference.

\section{Conclusions}

Occult MPCC is uncommon and complicated. MDCT is a leading diagnostic modality in which a communication between the cystic lesion and the biliary system is the key finding to specify the disease site. Although an optimal approach for occult MPCC has not been fully addressed, this case report provides a simple MDCT-based diagnostic approach and surgical management for this challenging scenario.

\section{Authors' contributions}

$M N, T E, Y Y, T I, G S, T M, J Y$, and MK performed the surgery and perioperative management on the patient. MN, TE, and MK drafted the manuscript. All authors read and approved the final manuscript.

\section{Competing interests}

The authors declare that they have no competing interests.

\section{Consent for publication}

Written informed consent was obtained from the patient for publication of this case report and any accompanying images. A copy of the written consent is available for review by the Editor-in-Chief of this journal.

Received: 31 August 2016 Accepted: 20 December 2016

Published online: 04 January 2017

\section{References}

1. Nagino M, Nimura Y, Hayakawa N, et al. A clinicopathological study of mucus producing bile duct carcinoma (in Japanese). Nihon geka gakkai Zasshi. 1990;91:695-704.

2. Kokubo T, Itai Y, Ohtomo K, et al. Mucin-hypersecreting Intrahepatic Biliary Neoplasm. Radiology. 1988;168:609-14.

3. Chen MF, Jan YY, Wang CS, et al. Clinical studies of mucin-producing cholangiocellular carcinoma. A study of 22 histopathology- proven cases. Ann Surg. 1998;227:63-9.

4. Sakamoto E, Nimura Y, Hayakawa N, et al. Clinicopathological studies of mucin-producing cholangiocarcinoma. J Hepatobiliary Pancreat Surg. 1997; 4:157-62. 
5. Sakamoto E, Hayakawa N, Junichi K, et al. Treatment strategy for mucinproducing intrhepatic cholangiocarcinoma: value of percutaneous transhepatic biliary drainage and cholangioscopy. World J Surg. 1999 ;23:1038-44.

6. Yang J, Wang W, Yan L. The clinicopathological features of intraductal papillary neoplasms of the bile duct in a Chinese population. Dig Liver Di. 2012;107:251-6

7. Lee SS, Kim MH, Lee SK, et al. Clinicopathologic review of 58 patients with biliary papillomatosis. Cancer. 2004;100:783-93.

8. Edge SB, Byrad DR, Compton CC, et al. AJCC Cancer Staging Manual. 7th ed. New York: Springer; 2010.

9. Chou ST, Chan CW. Mucin-producing cholangiocarcinoma: an autopsy study in Hong Kong. Pathology. 1976;8:321-8.

10. Chen TC, Nakamura Y, Zen Y, et al. Intraductal papillary neoplasia of the liver associated with hepatolithiasis. Hepatology. 2001;34:651-8.

11. Shibahara $\mathrm{H}$, Tamada S, Goto M, et al. Pathologic features of mucin-producing bile duct tumors: two histopathologic categories as counterparts of pancreatic intraductal papillary-mucinous neoplasms. Am J Surg Pathol. 2004;28:327-38.

12. Chen MF, Jan YY, Wang CS, et al. A reappraisal of cholangiocarcinoma in patient with hepatolithiasis. Cancer. 1993;71:2461-5.

13. Nakanuma Y, Sasaki M, Ishikawa A, et al. Biliary papillary neoplasm of the liver. Histol Histopathol. 2002:17:851-61.

14. Zen Y, Fujii T, Itatsu K, et al. Biliary papillary tumors share pathological features with intraductal papillary mucinous neoplasms of the pancreas. Hepatology. 2006;44:1333-43.

15. Onoe S, Shimoyama Y, Ebata T, et al. Clinicopathological significance of mucin production in patients with papillary cholangiocarcinoma. World J Surg. 2015;39:1177-84

16. Yeh TS, Tseng JH, Chiu CT, et al. Cholangiographic spectrum of intraductal papillary mucinous neoplasm of the bile ducts. Ann Surg. 2006;244:248-53.

17. Tsou YK, Liu NJ, Wu RC, et al. Endoscopic retrograde cholangiography in the diagnosis and treatment of mucobilia. Scand J Gastroenterol. 2008;43:1137-44.

18. Igami T, Nagino M, Oda K, et al. Clinicopathologic study of chlaongiocarcunoma with superficial spread. Ann Surg. 2009;249:296-302.

19. Tsukahara T, Ebata T, Shimoyama Y, et al. Residual carcinoma in situ at the ductal stump has a negative survival effect. Ann Surg 2016 ( in press.)

20. Nimura Y. Staging cholangiocarcinoma by cholangioscopy. HPB. 2008;10:113-5.

21. Tsuyuguchi T, Sasaki Y, Sugiyama H, et al. Endoscopic diagnosis of intraductal papillary mucinous neoplasm of the bile duct. J Hepatobiliary Pancreat Sci. 2010;17:230-5.

\section{Submit your manuscript to a SpringerOpen ${ }^{\circ}$ journal and benefit from:}

- Convenient online submission

- Rigorous peer review

- Immediate publication on acceptance

- Open access: articles freely available online

- High visibility within the field

- Retaining the copyright to your article 